\title{
Superstring Loop Amplitudes from the Field Theory Limit
}

\author{
Yvonne Geyer®* \\ Department of Physics, Faculty of Science, Chulalongkorn University, \\ Thanon Phayathai, Pathumwan, Bangkok 10330, Thailand \\ Ricardo Monteiro ${ }^{\dagger}$ and Ricardo Stark-Muchão \\ Centre for Research in String Theory, School of Physics and Astronomy, Queen Mary University of London, \\ E1 4NS, London, United Kingdom
}

(Received 23 August 2021; accepted 26 October 2021; published 16 November 2021)

\begin{abstract}
We propose a procedure to determine the moduli-space integrands of loop-level superstring amplitudes for massless external states in terms of the field theory limit. We focus on the type II superstring. The procedure is to (i) take a supergravity loop integrand written in a BCJ double-copy representation, (ii) use the loop-level scattering equations to translate that integrand into the ambitwistor string moduli-space integrand, localised on the nodal Riemann sphere, and (iii) uplift that formula to one on the higher-genus surface valid for the superstring, guided by modular invariance. We show how this works for the four-point amplitude at two loops, where we reproduce the known answer, and at three loops, where we present a conjecture that is consistent with a previous proposal for the chiral measure. Useful supergravity results are currently known up to five loops.
\end{abstract}

DOI: 10.1103/PhysRevLett.127.211603

Introduction.-The birth of string theory is widely considered to be the discovery by Veneziano of the scattering amplitude formula that today bears his name [1]. More than five decades later, the calculation of string scattering amplitudes remains a formidable challenge. To give the example of the type II superstring in Minkowski spacetime, the four-point amplitude for massless external states was computed at tree level and one loop in 1982 [2,3], and at two loops in 2005 [4-6]. There has been significant work on the three-loop problem, namely, a proposal for the chiral measure [7-9] and a partial computation using the pure spinor formalism [10], but it remains to be fully addressed. The advances have had a rich interplay with those in gauge theory and gravity amplitudes, particularly in their maximally supersymmetric versions. For instance, the first computations of the fourpoint one-loop amplitudes in the now widely studied 4D $\mathcal{N}=4$ super-Yang-Mills theory $(\mathrm{SYM})$ and $\mathcal{N}=8$ supergravity were based on the field theory limit of the analogous superstring calculations [11]. In this Letter, we aim to return the favor by importing three-loop results in $\mathcal{N}=8$ supergravity, themselves obtained from nonplanar

Published by the American Physical Society under the terms of the Creative Commons Attribution 4.0 International license. Further distribution of this work must maintain attribution to the author(s) and the published article's title, journal citation, and DOI. Funded by SCOAP .
$\mathcal{N}=4$ SYM via the Bern-Carrasco-Johansson (BCJ) double copy [12], into the type II superstring.

String theory versus field theory.-We will consider the type II superstring four-point amplitude for massless incoming states of momenta $k_{i}(i=1, \ldots, 4)$. The 10D maximal supersymmetry implies that information on the four external states is encoded in a kinematic prefactor $\mathcal{R}^{4}$ [13], such that the supergravity tree-level amplitude is $\sim \mathcal{R}^{4} /\left(s_{12} s_{13} s_{14}\right)$. We define the Mandelstam variables as $s_{i j}=2 k_{i} \cdot k_{j}$. Our working assumption will be that, up to three loops [14], the $g$-loop superstring amplitude $\mathcal{A}_{\mathbb{S}}^{(g)}$ takes the form

$$
\begin{aligned}
\frac{\mathcal{A}_{\mathbb{S}}^{(g)}}{\mathcal{R}^{4}}= & \int_{\mathcal{M}_{g, 4}}\left|\prod_{I \leq J} d \Omega_{I J}\right|^{2} \int d \ell\left|\mathcal{Y}_{\mathbb{S}}^{(g)}\right|^{2} \prod_{i<j}\left|E\left(z_{i}, z_{j}\right)\right|^{\alpha^{\prime} s_{i j}} \\
& \times\left|\exp \frac{\alpha^{\prime}}{2}\left(i \pi \Omega_{I J} \ell^{I} \cdot \ell^{J}+2 \pi i \sum_{j} \ell^{I} \cdot k_{j} \int_{z_{0}}^{z_{j}} \omega_{I}\right)\right|^{2} .
\end{aligned}
$$

The integration denoted by $\mathcal{M}_{g, 4}$ is over a genus- $g$ fundamental domain parametrized by the period matrix $\Omega_{I J}(I, J=1, \ldots, g)$ and over four marked points $z_{i}$. We use a "chiral splitting" representation [16,17], made possible by the introduction of the loop momenta $\ell^{I}$, with $d \ell$ denoting $\prod_{I} d^{10} \ell^{I}$. The appearance of the prime form $E\left(z_{i}, z_{j}\right)$ and the exponential (involving the holomorphic Abelian differentials $\omega_{I}$ whose cycles define the period matrix) constitute 
the chiral $\times$ antichiral loop-level Koba-Nielsen factors. The interesting object is $\mathcal{Y}_{\mathbb{S}}^{(g)}$. We make no distinction between type IIA and type IIB apart from the details of $\mathcal{R}^{4}$, since at four points there is no contribution from odd spin structures at least up to three loops [18].

We will exploit the analogy between the formula (1) for the superstring and the following expected formula for supergravity:

$\frac{\mathcal{A}_{\AA}^{(g)}}{\mathcal{R}^{4}}=\int d \ell \int_{\mathcal{M}_{g, 4}} \prod_{I \leq J} d \Omega_{I J}\left(\mathcal{Y}_{\AA}^{(g)}\right)^{2} \prod_{i=1}^{4} \bar{\delta}\left(\mathcal{E}_{i}\right) \prod_{I \leq J} \bar{\delta}\left(u^{I J}\right)$.

This type of formula for a scattering amplitude was discovered at tree level by Cachazo, He, and Yuan $[20,21]$, generalizing a previous formula from twistor string theory [22,23]. The loop-level extension [24-30] was derived from the type II ambitwistor string [31], which is a worldsheet model of type II supergravity. The 10D loop integration in Eq. (2) is UV divergent, so the expression is formal only, and we understand it as defining a loop integrand. The genus- $g$ moduli-space integration is fully localized on a set of critical points, determined by the genus- $g$ scattering equations: $\mathcal{E}_{i}=0$ and $u^{I J}=0$ [32]. An extensive discussion of the loop-level version of this formalism was presented in Ref. [30]; the brief discussion below will be sufficient for our purposes. There is a clear analogy between Eqs. (1) and (2). Our proposal, under conditions to be discussed, is to identify the "chiral halfintegrands,"

$$
\mathcal{Y}_{\mathbb{S}}^{(g)}=\mathcal{Y}_{\AA}^{(g)},
$$

which is known to be possible for $g \leq 2$. Notice that Eq. (1) is a simplified expression where $\mathcal{Y}_{\mathbb{S}}^{(g)}$ is independent of $\alpha^{\prime}$. The idea is that we can import an ambitwistor string-i.e., supergravity-result into the superstring.

The only known procedure to evaluate Eq. (2) reflects the fact that the ambitwistor string is a field theory in disguise: the genus- $g$ formula can be localized on a maximal nonseparating degeneration, i.e., a Riemann sphere with $g$ nodes, as in Fig. 1. This follows from a residue argument in moduli space at one $[27,28]$ and two $[29,30]$ loops, and our three-loop results provide evidence that it holds at higher order. The formula on the nodal sphere is

$\frac{\mathcal{A}_{\AA}^{(g)}}{\mathcal{R}^{4}}=\int \frac{d \ell}{\prod_{I}\left(\ell^{I}\right)^{2}} \int_{\mathcal{M}_{0,4+2 g}} c^{(g)}\left(\mathcal{J}^{(g)} \mathcal{Y}^{(g)}\right)^{2} \prod_{A=1}^{4+2 g} \bar{\delta}\left(\mathcal{E}_{A}\right)$.

Here, $\mathcal{M}_{0,4+2 g}$ is the moduli space of the Riemann sphere with $4+2 g$ marked points, corresponding to 4 external particles and $2 g$ "loop marked points," one pair per node as in Fig. 1. The factors $c^{(g)}$ and $\mathcal{J}^{(g)}$ arise from the
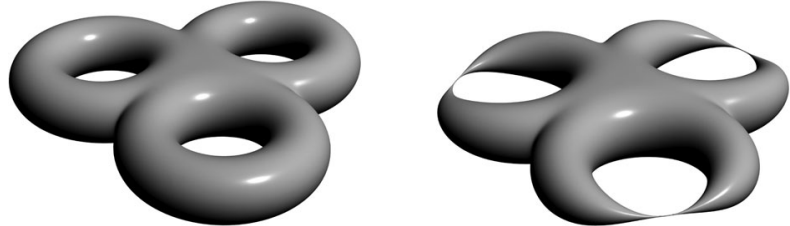

FIG. 1. Genus-3 surface and its maximal nonseparating degeneration (genus 0 ) with 2 marked points per node.

degeneration of $\mathcal{M}_{g, 4}$ to $\mathcal{M}_{0,4+2 g}$ [30]. We will give an example momentarily. The object $\mathcal{Y}^{(g)}$ in this expression is the limit of $\mathcal{Y}_{\mathrm{A}}^{(g)}$ in the maximal nonseparating degeneration. Finally, the delta functions impose the loop-level scattering equations on the nodal sphere, $\mathcal{E}_{A}=0$, on whose finite set of solutions the moduli-space integral fully localizes; in fact, this integral can be understood as a multidimensional residue integral.

Let us be more concrete. The degeneration to the $g$-nodal sphere is achieved in a limit involving the diagonal components of the period matrix: $q_{I I}=e^{i \pi \Omega_{I I}} \rightarrow 0$. In this limit, the holomorphic Abelian differentials whose periods define the period matrix acquire simple poles at the corresponding node: with $\sigma \in \mathbb{C P}^{1}$,

$$
\omega_{I}=\frac{\omega_{I^{+} I^{-}}}{2 \pi i}, \quad \omega_{I^{+} I^{-}}(\sigma)=\frac{\left(\sigma_{I^{+}}-\sigma_{I^{-}}\right) d \sigma}{\left(\sigma-\sigma_{I^{+}}\right)\left(\sigma-\sigma_{I^{-}}\right)},
$$

where the $\sigma_{I^{ \pm}}$are the marked points for node $I$. Together with the marked points $\sigma_{i}$ associated to the four external particles, we have the total of $4+2 g$ marked points parametrizing $\mathcal{M}_{0,4+2 g}$ up to $\operatorname{SL}(2, \mathbb{C})$. For $g \geq 2$, the off-diagonal components of the period matrix are expressed in this limit in terms of cross ratios of the nodal marked points,

$$
q_{I J}=e^{2 i \pi \Omega_{I J}}=\frac{\sigma_{I^{+} J^{+}} \sigma_{I^{-} J^{-}}}{\sigma_{I^{+} J^{-}} \sigma_{I^{-} J^{+}}},
$$

where we denote $\sigma_{A B}=\sigma_{A}-\sigma_{B}$. This change of integration variables leads to the $\left(\mathcal{J}^{(g)}\right)^{2}$ appearing in Eq. (4). One $\mathcal{J}^{(g)}$ arises from the moduli-space measure,

$\prod_{I<J} \frac{d q_{I J}}{q_{I J}}=\frac{\mathcal{J}^{(g)}}{\operatorname{vol} \operatorname{SL}(2, \mathbb{C})}, \quad \mathcal{J}^{(g)}=J^{(g)} \prod_{I^{ \pm}} d \sigma_{I^{ \pm}}$,

while the other arises from rewriting higher-genus scattering equations as nodal sphere ones. Finally, the scattering equations on the nodal sphere are equivalent to the vanishing of a meromorphic quadratic differential $\mathfrak{P}^{(g)}$ with only simple poles, and can be read off from the residues of this differential at the $4+2 g$ marked points, 


$$
\mathcal{E}_{A}=\operatorname{Res}_{\sigma_{A}} \mathfrak{P}^{(g)} .
$$

The ingredients of Eq. (4) can be illustrated with the twoloop example. We have $c^{(2)}=1 /\left(1-q_{12}\right)$ [33] and

$$
\mathfrak{P}^{(2)}=P^{2}-\left(\ell^{I} \omega_{I^{+} I^{-}}\right)^{2}+\left(\ell_{1}^{2}+\ell_{2}^{2}\right) \omega_{1^{+} 1^{-}} \omega_{2^{+} 2^{-}},
$$

where

$$
P_{\mu}(\sigma)=\ell_{\mu}^{I} \omega_{I^{+} I^{-}}(\sigma)+\sum_{i} \frac{k_{i \mu}}{\sigma-\sigma_{i}} d \sigma
$$

Effectively, $\mathfrak{P}^{(g)}$ encodes all the potential loop-integrand propagators in an expression like Eq. (4), while $c^{(g)}$ projects out certain unphysical propagators. These details are not important for this Letter, where we are concerned with $\mathcal{J}^{(g)}$ and especially $\mathcal{Y}^{(g)}$. At two loops, we have

$$
J^{(2)}=\frac{1}{\sigma_{1^{+} 2^{+}} \sigma_{1^{+} 2^{-}} \sigma_{1^{-} 2^{+}} \sigma_{1^{-} 2^{-}}}
$$

and

$$
\mathcal{Y}^{(2)}=\frac{1}{3}\left[\left(s_{14}-s_{13}\right) \Delta_{12}^{(2)} \Delta_{34}^{(2)}+\operatorname{cyc}(234)\right],
$$

where we used the determinant

$$
\Delta_{i_{1} \ldots i_{g}}^{(g)}=\varepsilon^{I_{1} \ldots I_{g}} \omega_{I_{1}}\left(\sigma_{i_{1}}\right) \ldots \omega_{I_{g}}\left(\sigma_{i_{g}}\right)
$$

defined for any $g$. The expression (12) is built from the differentials $\omega_{I}$, which naturally lift from the nodal sphere to become the holomorphic Abelian differentials on the genus-2 surface. Indeed, the genus-2 expression is also valid as $\mathcal{Y}_{\AA}^{(2)}$ in Eq. (2) and, crucially for us, as $\mathcal{Y}_{\S}^{(2)}$ in Eq. (1). The object $\Delta^{(g)}$ is a modular form of weight -1 at any genus, which at genus 2 gives $\mathcal{Y}_{\mathbb{S}}^{(2)}$ the appropriate weight such that the moduli-space integral is well defined. At three loops, the answer is not as simple as Eq. (12): $\Delta^{(3)}$ still arises [10], but additional ingredients are needed, as discussed, e.g., in Ref. [34], and as we will see here.

$\mathcal{Y}_{\mathbb{S}}^{(g)}$ from BCJ numerators. - Let us present and test our strategy. The steps are to (i) take a supergravity loop integrand written in a BCJ double-copy representation, (ii) translate that integrand into the ambitwistor string moduli-space integrand localized on the nodal Riemann sphere, i.e., obtain $\mathcal{Y}^{(g)}$, (iii) uplift that formula to a highergenus modular form conjecturally valid for the superstring, i.e., obtain $\mathcal{Y}_{\mathbb{S}}^{(g)}$ such that $\mathcal{Y}_{\mathbb{S}}^{(g)} \rightarrow \mathcal{Y}^{(g)}$ as $q_{I I} \rightarrow 0$. With our current understanding, step (iii) relies on an educated guess, as we will exemplify.

Starting with step (i), a BCJ representation is one in which the loop integrand is written in terms of trivalent diagrams, whose numerators are the square of analogous numerators in nonplanar SYM obeying the BCJ colorkinematics duality [12,35] [36]. See Ref. [47] for a review of this remarkable construction, which was motivated by the KLT relations of string theory [48]. Indeed, there is a large body of work relating this construction to aspects of string theory, e.g., Refs. [49-65]. Step (ii) is based on the connection to the scattering equations story, for which we use the following relation based on a differential form with logarithmic singularities [66]

$$
(2 \pi i)^{4} \mathcal{J}^{(g)} \mathcal{Y}^{(g)}=\sum_{\rho \in S_{2+2 g}} \frac{N^{(g)}\left(1^{+}, \rho, 1^{-}\right)}{\left(1^{+}, \rho, 1^{-}\right)} \prod_{A=1}^{4+2 g} d \sigma_{A},
$$

where $(A B C \ldots D)=\sigma_{A B} \sigma_{B C} \ldots \sigma_{D A}$ is a Parke-Taylor denominator. The BCJ numerators $N^{(g)}$, which depend on a particle ordering, are SYM numerators whose square gives the supergravity numerators; this square effectively translates into the square of $\mathcal{J}^{(g)} \mathcal{Y}^{(g)}$ in Eq. (4). Notice, however, that we have extracted the overall factor $\mathcal{R}^{4}$ in Eq. (4), whose "square root" is therefore not included in the SYM numerators. The correspondence between the numerators $N^{(g)}$ and trivalent diagrams is best understood in an explicit example, to be discussed below. Before that, let us make two comments. The first is that two marked points singled out in Eq. (14) were chosen to be $\sigma_{1^{ \pm}}$, but the sum is independent of that choice. The second, for the reader familiar with the scattering equations formalism including the developments [70-73], is that equalities like (14) often hold only when the marked points satisfy the scattering equations (e.g., for CHY Pfaffians). Here, on the other hand, we propose that Eq. (14) defines $\mathcal{Y}^{(g)}$ such that it may be uplifted to the superstring, as happens up to two loops.

Let us test the strategy at two loops, for which the BCJ representation of the four-point supergravity loop integrand is long known [74,75]. The two-loop BCJ numerators can be compactly written as

$$
N^{(2)}\left(1^{+}, \rho_{1}, 2^{ \pm}, \rho_{2}, 2^{\mp}, \rho_{3}, 1^{-}\right)= \begin{cases}s_{i j} & \rho_{2}=\{i, j\} \\ 0 & \text { otherwise }\end{cases}
$$

They correspond to half-ladder diagrams with loop momenta $\pm \ell_{1}$ at the ends; see Fig. 2. A standard twoloop diagram is then obtained by gluing the nodal legs, i.e., $I^{+}$with $I^{-}$. Taking the result (15) from the literature, it is possible to obtain $\mathcal{Y}^{(2)}$ via Eq. (14). Then, it is both natural

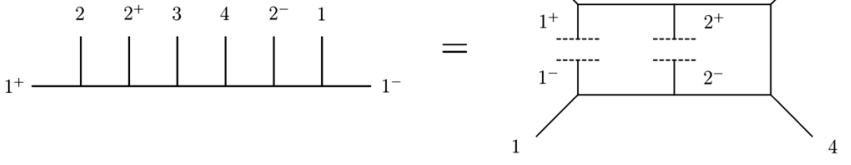

FIG. 2. Two-loop example. Diagram associated with the numerator $N\left(1^{+}, 2,2^{+}, 3,4,2^{-}, 1,1^{-}\right)$. 
and easy to rewrite $\mathcal{Y}^{(2)}$ in the form (12), which, as explained earlier, can be uplifted to genus 2, matching the superstring result $\mathcal{Y}_{\mathbb{S}}^{(2)}$. This achieves step (iii).

Three loops.-We now apply our strategy to the much more intricate three-loop case. From the general form of a three-loop field theory integrand, namely, the inclusion of the relevant diagram topologies, we can determine $c^{(3)}$ and $\mathfrak{P}^{(3)}$. However, they do not appear in Eq. (14), so they are not important for the goal of this Letter [76]. The important quantities are $\mathcal{J}^{(3)}$ and $\mathcal{Y}^{(3)}$. The Jacobian is straightforwardly obtained from Eq. (7) and can be written as

$$
J^{(3)}=J_{\text {hyp }} \frac{\prod_{I} \sigma_{I^{+} I^{-}}}{\prod_{I<J} \sigma_{I^{+} J^{+}} \sigma_{I^{-} J^{-}} \sigma_{I^{+} J^{-}} \sigma_{I^{-} J^{+}}},
$$

where in the factor

$$
J_{\text {hyp }}=\sigma_{1^{+} 2^{-}} \sigma_{2^{+} 3^{-}} \sigma_{3^{+} 1^{-}}-\sigma_{1^{+} 3^{-}} \sigma_{3^{+} 2^{-}} \sigma_{2^{+} 1^{-}}
$$

the subscript refers to hyperelliptic, as we will explain.

We can now determine $\mathcal{Y}^{(3)}$ using Eq. (14). The righthand side is obtained from the known BCJ representation of the three-loop supergravity integrand, a landmark application of the double copy [12,77]. The BCJ numerators, listed in Table I of Ref. [12], are not as simple as at two loops and depend linearly on the loop momenta, e.g., [78]

$$
N\left(1^{+}, 1,2,2^{+}, 3,3^{+}, 2^{-}, 4,3^{-}, 1^{-}\right)=\frac{1}{3} s_{12}\left(s_{12}-s_{14}\right)+\frac{2}{3} \ell^{1} \cdot\left[k_{2}\left(s_{13}-s_{14}\right)+k_{3}\left(s_{13}-s_{12}\right)+k_{4}\left(s_{12}-s_{14}\right)\right] .
$$

Via Eq. (14), this property implies

$$
2 \pi i \mathcal{Y}_{\mathbb{S}}^{(3)}=\mathcal{Y}_{0}+2 \pi i \ell_{\mu}^{I} \mathcal{Y}_{I}^{\mu}
$$

where the factors were chosen for later convenience. We write our results already in uplifted form, i.e., for $\mathcal{Y}_{\mathbb{S}}^{(3)}$ (which we claim is $\mathcal{Y}_{A}^{(3)}$ ) instead of its degeneration $\mathcal{Y}^{(3)}$. To determine $\mathcal{Y}_{\mathbb{S}}^{(3)}$, we construct a well-motivated ansatz with the required modular weight of -1 , and fix the coefficients of that ansatz by matching numerically the degeneration limit to Eq. (14). This requires expanding in the degeneration parameters the Jacobi theta functions which define various objects, a straightforward if computationally heavy procedure.

The second term in Eq. (18) is the easiest: we can write

$$
\mathcal{Y}_{I}^{\mu}=\frac{2}{3}\left[\alpha_{1}^{\mu} \omega_{I}\left(z_{1}\right) \Delta_{234}^{(3)}+\operatorname{cyc}(1234)\right],
$$

with $\alpha_{1}^{\mu}=k_{2}^{\mu}\left(k_{3}-k_{4}\right) \cdot k_{1}+\operatorname{cyc}(234)$. All the ingredients have been introduced previously.

The object $\mathcal{Y}_{0}$ is more involved. It is convenient to extricate the kinematic dependence by writing

$$
\mathcal{Y}_{0}=s_{13} s_{14} Y_{12,34}+\operatorname{cyc}(234) \text {, }
$$

where $Y_{12,34}$ is independent of the $s_{i j}$ and is symmetric when exchanging: $z_{1} \leftrightarrow z_{2}, z_{3} \leftrightarrow z_{4},\left\{z_{1}, z_{2}\right\} \leftrightarrow\left\{z_{3}, z_{4}\right\}$. Let us first state the result and then discuss it:

$$
Y_{12,34}=\frac{1}{3} \mathcal{D}_{12,34}-\frac{1}{15 \Psi_{9}}\left(\mathcal{S}_{12,34}^{(a)}-\frac{1}{8} \mathcal{S}_{12,34}^{(b)}\right),
$$

where

$$
\begin{gathered}
\mathcal{D}_{12,34}=\omega_{3,4}\left(z_{1}\right) \Delta_{234}^{(3)}+\omega_{3,4}\left(z_{2}\right) \Delta_{134}^{(3)} \\
+\omega_{1,2}\left(z_{3}\right) \Delta_{412}^{(3)}+\omega_{1,2}\left(z_{4}\right) \Delta_{312}^{(3)} \\
\mathcal{S}_{12,34}^{(a)}=\sum_{\delta} \Xi_{8}[\delta]\left[S_{\delta}\left(z_{1}, z_{2}\right) S_{\delta}\left(z_{2}, z_{3}\right) S_{\delta}\left(z_{3}, z_{4}\right) S_{\delta}\left(z_{4}, z_{1}\right)\right. \\
\left.+S_{\delta}\left(z_{2}, z_{1}\right) S_{\delta}\left(z_{1}, z_{3}\right) S_{\delta}\left(z_{3}, z_{4}\right) S_{\delta}\left(z_{4}, z_{2}\right)\right] \\
\mathcal{S}_{12,34}^{(b)}=\sum_{\delta} \Xi_{8}[\delta] S_{\delta}\left(z_{1}, z_{2}\right)^{2} S_{\delta}\left(z_{3}, z_{4}\right)^{2}
\end{gathered}
$$

Starting with the expression (22), the object $\omega_{i, j}\left(z_{k}\right)$ is the normalized Abelian differential of the third kind, whose degeneration limit is

$$
\omega_{i, j}(\sigma)=\frac{\sigma_{i j}}{\left(\sigma-\sigma_{i}\right)\left(\sigma-\sigma_{j}\right)} d \sigma .
$$

A consistency check is that the contribution (22), including the kinematic coefficient, is completely fixed by Eq. (19). This follows from the condition of "homology invariance": distinct choices of homology cycles of the Riemann surface with respect to the marked points $z_{i}$ obey monodromy relations dictated by the chiral splitting procedure [17], and this connects the two contributions [79].

The contributions (23) and (24) are more elaborate, but the structure is familiar from the RNS formalism $[4,16,81-86]$. The sums are over the 36 even spin structures at genus 3, labeled by $\delta$, and the objects $S_{\delta}\left(z_{i}, z_{j}\right)$ are the Szegó kernels arising from the OPEs of worldsheet fermions. The "chiral measure" $\Xi_{8}[\delta] / \Psi_{9}$ is the crucial ingredient. Here, $\Psi_{9}=\sqrt{-\prod_{\delta} \theta[\delta](0)}$ is a modular form of weight 9 (note our nonstandard definition for the sign), defined in terms of the even Jacobi theta functions. 
The general properties of the chiral measure were described in Refs. $[7,8]$ and the precise definition of $\Xi_{8}[\delta]$ was given in Ref. [9]. It is a sophisticated definition, so we will not repeat it here; we found Ref. [87] very helpful. The RNS derivation of this measure remains obscure; see Appendix C of Ref. [88].

In the degeneration limit $q_{I I} \rightarrow 0, \Psi_{9}$ vanishes with leading behavior $\Psi_{9}=\left(\prod_{I} q_{I I}^{2}\right) \psi_{9}+\cdots$,

$$
\psi_{9}=2^{14} J_{\mathrm{hyp}} \frac{\left(\prod_{I} \sigma_{I^{+} I^{-}}\right)^{3}}{\prod_{I<J} \sigma_{I^{+} J^{+}} \sigma_{I^{-} J^{-}} \sigma_{I^{+} J^{-}} \sigma_{I^{-} J^{+}}},
$$

where $J_{\text {hyp }}$ is given in Eq. (17). It is opportune to note that only a codimension- $1_{\mathbb{C}}$ subset of genus-3 Riemann surfaces are hyperelliptic (whereas for $g \leq 2$ all surfaces are), and these are precisely identified by the vanishing of $\Psi_{9}$ [89]. The condition $J_{\text {hyp }}=0$ identifies hyperelliptic surfaces in the degeneration limit. The factors of $J_{\text {hyp }}$ in $J^{(3)}$ and in $1 / \Psi_{9}$ cancel, such that $\mathcal{J}^{(3)} \mathcal{Y}^{(3)}$ does not vanish in the hyperelliptic sector.

The sums (23) and (24), which are modular forms of weight 8 , vanish in the degeneration limit in a manner analogous to $\Psi_{9}$, so that the ratio appearing in Eq. (21) yields a finite result on the nodal sphere [91]. As consistency checks on our implementation of the chiral measure, we verified to order $O\left(q_{I I}^{2}\right)$ the following identities (respectively, from Refs. [9,92,93]):

$$
\begin{aligned}
& \sum_{\delta} \Xi_{8}[\delta]=0, \quad \sum_{\delta} \Xi_{8}[\delta] S_{\delta}\left(z_{1}, z_{2}\right)^{2}=0, \\
& \sum_{\delta} \Xi_{8}[\delta] S_{\delta}\left(z_{1}, z_{2}\right) S_{\delta}\left(z_{2}, z_{3}\right) S_{\delta}\left(z_{3}, z_{1}\right)=C \Psi_{9} \Delta_{123}^{(3)},
\end{aligned}
$$

where we determined the previously unknown coefficient $C=15(2 \pi i)^{3}$. We could not find simplified expressions for Eqs. (23) and (24); they are not proportional to $\Psi_{9}$, i.e., not proportional to $J_{\text {hyp }}$ in the degeneration limit.

Comparing our result to the pure spinor computation of Ref. [10], the latter was restricted to part of the correlator and was not manifestly modular invariant, but appears to be consistent at least with Eq. (19). The main goal of Ref. [10], for which the partial computation was sufficient, was to match a prediction from $S$ duality [94] for the low-energy amplitude, where the overall normalization is important. We neglected the normalization here, and leave this aspect and a proper comparison to Ref. [10] for future work. Because of manifest supersymmetry, the splitting of spin structures does not arise in the pure spinor approach [5,6,95-98], so this approach may be helpful in simplifying the sums seen above.

Discussion.-We have constructed a conjectured expression for the three-loop four-point amplitude of massless states in the type II superstring. The crucial ingredient is the chiral half-integrand (18). As at two loops
$[4,80]$, this object can also, in principle, be imported into the Heterotic superstring, paired with a bosonic counterpart.

In place of a first-principles worldsheet calculation, we wrote down an ansatz inspired by insights from the RNS and pure spinor formalisms, and then constrained that ansatz using supergravity data mined with modern amplitudes techniques. Our focus was on briefly delineating a strategy, with very concrete results. Additional technical details will be presented elsewhere. We hope that our conjecture can guide rigorous derivations using established worldsheet methods. Alternatively, in the spirit of the amplitudes program, perhaps the proof can follow from a set of basic constraints, such as unitarity.

Natural future directions are the study of the modulispace integration in the low-energy limit, building on Refs. [10,99-102], which is newly motivated by beautiful advances in the nonperturbative amplitudes bootstrap [103]; and the consideration of higher-point [7,9,112-118] amplitudes. We expect our strategy to prove useful, not least because there are BCJ numerators for $\mathcal{N}=8$ supergravity up to five loops [119-121], although the five-loop case required a generalization of this representation. Also at this loop order, the relation between supermoduli space and ordinary moduli space becomes more intricate [15], calling into question the structure of our starting point (1). The interplay between field theory and string theory amplitudes continues to present us with many challenges and fruitful surprises.

We thank Eric D'Hoker, Carlos Mafra, Boris Pioline, Rodolfo Russo, Oliver Schlotterer, and Edward Witten for comments. Y. G. is supported by the CUniverse research promotion project "Toward World-class Fundamental Physics" of Chulalongkorn University (Grant No. CUAASC). R. M. and R. S. M. are supported by the Royal Society via a University Research Fellowship and a Studentship Grant, respectively.

Note added.-Recently, it came to our attention that the authors of Ref. [80] have independently constructed the contribution to the half-integrand that is linear in the loop momenta, Eq. (19).

*yjgeyer@gmail.com

†ricardo.monteiro@qmul.ac.uk

†r.j.stark-muchao@qmul.ac.uk

[1] G. Veneziano, Construction of a crossing-symmetric, Regge behaved amplitude for linearly rising trajectories, Nuovo Cimento A 57, 190 (1968).

[2] M. B. Green and J. H. Schwarz, Supersymmetrical string theories, Phys. Lett. 109B, 444 (1982).

[3] J. H. Schwarz, Superstring theory, Phys. Rep. 89, 223 (1982). 
[4] E. D'Hoker and D. H. Phong, Two-loop superstrings VI: Non-renormalization theorems and the 4-point function, Nucl. Phys. B715, 3 (2005).

[5] N. Berkovits, Super-poincare covariant two-loop superstring amplitudes, J. High Energy Phys. 01 (2006) 005.

[6] N. Berkovits and C. R. Mafra, Equivalence of Two-Loop Superstring Amplitudes in the Pure Spinor and RNS Formalisms, Phys. Rev. Lett. 96, 011602 (2006).

[7] E. D'Hoker and D. H. Phong, Asyzygies, modular forms, and the superstring measure II, Nucl. Phys. B710, 83 (2005).

[8] E. D'Hoker and D. H. Phong, Asyzygies, modular forms, and the superstring measure. I., Nucl. Phys. B710, 58 (2005).

[9] S. L. Cacciatori, F. Dalla Piazza, and B. van Geemen, Modular forms and three loop superstring amplitudes, Nucl. Phys. B800, 565 (2008).

[10] H. Gomez and C. R. Mafra, The closed-string 3-loop amplitude and S-duality, J. High Energy Phys. 10 (2013) 217.

[11] M. B. Green, J. H. Schwarz, and L. Brink, $N=4$ YangMills and $N=8$ supergravity as limits of string theories, Nucl. Phys. B198, 474 (1982).

[12] Z. Bern, J. J. M. Carrasco, and H. Johansson, Perturbative Quantum Gravity as a Double Copy of Gauge Theory, Phys. Rev. Lett. 105, 061602 (2010).

[13] M. B. Green, J. H. Schwarz, and E. Witten, Superstring Theory. Vol. 1: Introduction, Cambridge Monographs on Mathematical Physics (Cambridge University Press, Cambridge, England, 1988).

[14] Beyond three loops, the integration over the period matrix $\Omega_{I J}$ must be restricted due to the Schottky problem. Beyond four loops, the delicate issue of non-projectedness of supermoduli space is also known to arise [15].

[15] R. Donagi and E. Witten, Supermoduli space is not projected, Proc. Symp. Pure Math. 90, 19 (2015).

[16] E. D'Hoker and D. H. Phong, The geometry of string perturbation theory, Rev. Mod. Phys. 60, 917 (1988).

[17] E. D'Hoker and D. H. Phong, Conformal scalar fields and chiral splitting on superriemann surfaces, Commun. Math. Phys. 125, 469 (1989).

[18] This follows from supersymmetry and is clear if we consider factorizable NS-NS external states $\varepsilon_{i}^{\mu \nu}=\epsilon_{i}^{\mu} \tilde{\epsilon}_{i}^{\nu}$. The supersymmetric prefactor is $\mathcal{R}^{4}(\epsilon, \tilde{\epsilon})=\mathcal{F}^{4}(\epsilon) \mathcal{F}^{4}(\tilde{\epsilon})$, where $\mathcal{F}^{4}$ is the prefactor for the open superstring and includes products $\epsilon_{i} \cdot \epsilon_{j}$. At three loops and four points, a 10D Levi-Civita tensor arising from an odd spin structure's zero mode may just about be saturated: $\varepsilon_{10}\left(k_{1}, k_{2}, k_{3}\right.$, $\left.\epsilon_{1}, \epsilon_{2}, \epsilon_{3}, \epsilon_{4}, \ell_{1}, \ell_{2}, \ell_{3}\right)$, but it would never give rise to any $\epsilon_{i} \cdot \epsilon_{j}$. Moreover, the contraction of the two Levi-Civita tensors (with $\epsilon_{i}$ and with $\tilde{\epsilon}_{i}$ ) over three indices after loop integration, required for a potentially nonvanishing contribution, yields products $\epsilon_{i} \cdot \tilde{\epsilon}_{j}$, inconsistent with $\mathcal{F}^{4}(\epsilon) \mathcal{F}^{4}(\tilde{\epsilon})$. This discussion is consistent with the results of Refs. [10,19].

[19] N. Berkovits, New Higher-Derivative $R^{4}$ Theorems, Phys. Rev. Lett. 98, 211601 (2007).

[20] F. Cachazo, S. He, and E. Y. Yuan, Scattering of Massless Particles in Arbitrary Dimensions, Phys. Rev. Lett. 113, 171601 (2014).
[21] F. Cachazo, S. He, and E. Y. Yuan, Scattering of massless particles: Scalars, gluons and gravitons, J. High Energy Phys. 07 (2014) 033.

[22] E. Witten, Perturbative gauge theory as a string theory in twistor space, Commun. Math. Phys. 252, 189 (2004).

[23] R. Roiban, M. Spradlin, and A. Volovich, On the tree level S matrix of Yang-Mills theory, Phys. Rev. D 70, 026009 (2004).

[24] T. Adamo, E. Casali, and D. Skinner, Ambitwistor strings and the scattering equations at one loop, J. High Energy Phys. 04 (2014) 104.

[25] E. Casali and P. Tourkine, Infrared behaviour of the oneloop scattering equations and supergravity integrands, J. High Energy Phys. 04 (2015) 013.

[26] T. Adamo and E. Casali, Scattering equations, supergravity integrands, and pure spinors, J. High Energy Phys. 05 (2015) 120.

[27] Y. Geyer, L. Mason, R. Monteiro, and P. Tourkine, Loop Integrands for Scattering Amplitudes from the Riemann Sphere, Phys. Rev. Lett. 115, 121603 (2015).

[28] Y. Geyer, L. Mason, R. Monteiro, and P. Tourkine, Oneloop amplitudes on the Riemann sphere, J. High Energy Phys. 03 (2016) 114.

[29] Y. Geyer, L. Mason, R. Monteiro, and P. Tourkine, Twoloop scattering amplitudes from the Riemann sphere, Phys. Rev. D 94, 125029 (2016).

[30] Y. Geyer and R. Monteiro, Two-loop scattering amplitudes from ambitwistor strings: from genus two to the nodal Riemann sphere, J. High Energy Phys. 11 (2018) 008.

[31] L. Mason and D. Skinner, Ambitwistor strings and the scattering equations, J. High Energy Phys. 07 (2014) 048.

[32] For the delta functions, we use the definition $\bar{\delta}(z)=$ $\bar{\partial}(1 / 2 \pi i z)$, standard in this context.

[33] $c^{(2)}$ is associated to the genus-2 fundamental domain constraint $\left|q_{12}\right|<1$. In particular, $c^{(2)}$ arises from relaxing this constraint when degenerating to the sphere [30].

[34] P. Tourkine, Tropical amplitudes, Ann. Inst. Henri Poincaré 18, 2199 (2017).

[35] Z. Bern, J. J. M. Carrasco, and H. Johansson, New relations for gauge-theory amplitudes, Phys. Rev. D 78, 085011 (2008).

[36] It should be noted that the supergravity loop integrand in Eq. (4), once the moduli-space integral is performed, is not written in terms of Feynman-like propagators, as in the original BCJ representation [12]. It is instead written in an alternative representation, which was discovered in the ambitwistor string; see Refs. [27,28,37-46] for discussions. The supergravity BCJ numerators relevant in our two- and three-loop problems are valid in both representations.

[37] S. He and E. Y. Yuan, One-loop scattering equations and amplitudes from forward limit, Phys. Rev. D 92, 105004 (2015).

[38] C. Baadsgaard, N. E. J. Bjerrum-Bohr, J. L. Bourjaily, S. Caron-Huot, P. H. Damgaard, and B. Feng, New Representations of the Perturbative S-Matrix, Phys. Rev. Lett. 116, 061601 (2016).

[39] F. Cachazo, S. He, and E. Y. Yuan, One-loop corrections from higher dimensional tree amplitudes, J. High Energy Phys. 08 (2016) 008. 
[40] S. He and O. Schlotterer, New Relations for Gauge-Theory and Gravity Amplitudes at Loop Level, Phys. Rev. Lett. 118, 161601 (2017).

[41] B. Feng, CHY-construction of planar loop integrands of cubic scalar theory, J. High Energy Phys. 05 (2016) 061.

[42] S. He, O. Schlotterer, and Y. Zhang, New BCJ representations for one-loop amplitudes in gauge theories and gravity, Nucl. Phys. B930, 328 (2018).

[43] Y. Geyer and R. Monteiro, Gluons and gravitons at one loop from ambitwistor strings, J. High Energy Phys. 03 (2018) 068.

[44] Y. Geyer, R. Monteiro, and R. Stark-Muchão, Twoloop scattering amplitudes: double-forward limit and colour-kinematics duality, J. High Energy Phys. 12 (2019) 049.

[45] A. Edison, S. He, O. Schlotterer, and F. Teng, One-loop correlators and BCJ numerators from forward limits, J. High Energy Phys. 09 (2020) 079.

[46] J. A. Farrow, Y. Geyer, A. E. Lipstein, R. Monteiro, and R. Stark-Muchão, Propagators, BCFW recursion and new scattering equations at one loop, J. High Energy Phys. 10 (2020) 074.

[47] Z. Bern, J. J. Carrasco, M. Chiodaroli, H. Johansson, and R. Roiban, The duality between color and kinematics and its applications, arXiv:1909.01358.

[48] H. Kawai, D. C. Lewellen, and S. H. H. Tye, A relation between tree amplitudes of closed and open strings, Nucl. Phys. B269, 1 (1986).

[49] N. E. J. Bjerrum-Bohr, P. H. Damgaard, and P. Vanhove, Minimal Basis for Gauge Theory Amplitudes, Phys. Rev. Lett. 103, 161602 (2009).

[50] S. Stieberger, Open \& closed vs pure open string disk amplitudes, arXiv:0907.2211.

[51] C. R. Mafra, O. Schlotterer, and S. Stieberger, Explicit BCJ numerators from pure spinors, J. High Energy Phys. 07 (2011) 092.

[52] C. R. Mafra and O. Schlotterer, The structure of n-point one-loop open superstring amplitudes, J. High Energy Phys. 08 (2014) 099.

[53] A. Ochirov and P. Tourkine, BCJ duality and double copy in the closed string sector, J. High Energy Phys. 05 (2014) 136.

[54] C. R. Mafra and O. Schlotterer, Towards one-loop SYM amplitudes from the pure spinor BRST cohomology, Fortschr. Phys. 63, 105 (2015).

[55] S. He, R. Monteiro, and O. Schlotterer, String-inspired BCJ numerators for one-loop MHV amplitudes, J. High Energy Phys. 01 (2016) 171.

[56] C. R. Mafra and O. Schlotterer, Berends-Giele recursions and the BCJ duality in superspace and components, J. High Energy Phys. 03 (2016) 097.

[57] P. Tourkine and P. Vanhove, Higher-Loop Amplitude Monodromy Relations in String and Gauge Theory, Phys. Rev. Lett. 117, 211601 (2016).

[58] S. Hohenegger and S. Stieberger, Monodromy relations in higher-loop string amplitudes, Nucl. Phys. B925, 63 (2017).

[59] A. Ochirov, P. Tourkine, and P. Vanhove, One-loop monodromy relations on single cuts, J. High Energy Phys. 10 (2017) 105.
[60] P. Tourkine, Integrands and loop momentum in string and field theory, Phys. Rev. D 102, 026006 (2020).

[61] S. Mizera, Aspects of scattering amplitudes and moduli space localization, Ph.D. thesis, Princeton, Inst. Advanced Study, 2020.

[62] E. Casali, S. Mizera, and P. Tourkine, Monodromy relations from twisted homology, J. High Energy Phys. 12 (2019) 087.

[63] E. Casali, S. Mizera, and P. Tourkine, Loop amplitudes monodromy relations and color-kinematics duality, J. High Energy Phys. 03 (2021) 048.

[64] L. Borsten, H. Kim, B. Jurčo, T. Macrelli, C. Saemann, and M. Wolf, Double copy from homotopy algebras, Fortschr. Phys. 69, 2100075 (2021).

[65] E. Bridges and C. R. Mafra, Local BCJ numerators for tendimensional SYM at one loop, J. High Energy Phys. 07 (2021) 031.

[66] The tree-level $\left(\mathcal{J}^{(0)}=1\right)$ version of this relation was revealed in string theory in Refs. [51,67] and in the scattering equations (CHY) formalism in Ref. [21]; see also Refs. [68,69]. Our higher-loop formula is motivated by its verification at two loops in Ref. [44]. The highermultiplicity extension is trivial.

[67] C. R. Mafra, O. Schlotterer, and S. Stieberger, Complete $\mathrm{N}$-point superstring disk amplitude I. Pure spinor computation, Nucl. Phys. B873, 419 (2013).

[68] T. Azevedo, M. Chiodaroli, H. Johansson, and O. Schlotterer, Heterotic and bosonic string amplitudes via field theory, J. High Energy Phys. 10 (2018) 012.

[69] S. He, F. Teng, and Y. Zhang, String Amplitudes from Field-Theory Amplitudes and Vice Versa, Phys. Rev. Lett. 122, 211603 (2019).

[70] N. E. J. Bjerrum-Bohr, P. H. Damgaard, P. Tourkine, and P. Vanhove, Scattering equations and string theory amplitudes, Phys. Rev. D 90, 106002 (2014).

[71] S. Mizera, Kinematic Jacobi Identity is a Residue Theorem: Geometry of Color-Kinematics Duality for Gauge and Gravity Amplitudes, Phys. Rev. Lett. 124, 141601 (2020).

[72] N. Kalyanapuram, Ambitwistor integrands from tensionless chiral superstring integrands, J. High Energy Phys. 10 (2021) 171.

[73] N. Kalyanapuram, On chiral splitting and the ambitwistor string, Phys. Rev. D 104, 086027 (2021).

[74] Z. Bern, L. J. Dixon, D. C. Dunbar, M. Perelstein, and J. S. Rozowsky, On the relationship between Yang-Mills theory and gravity and its implication for ultraviolet divergences, Nucl. Phys. B530, 401 (1998).

[75] The known result is for $4 \mathrm{D} \mathcal{N}=8$ supergravity. The 10D type II supergravity amplitude is not defined, due to the UV divergence, but the loop integrand can be taken to be a straightforward dimensional "oxidation," with appropriate prefactor $\mathcal{R}^{4}$.

[76] They will be discussed elsewhere.

[77] The result in Ref. [12] applies to $4 \mathrm{D} \mathcal{N}=8$ supergravity, but we will assume that it "oxidates" trivially to 10D type II supergravity for similar reasons as in the two loop case, given the absence of contributions from odd spin structures. 
[78] Our convention for the external momenta is that they are incoming, whereas the convention in Ref. [12] was that they are outgoing. This affects the sign of the term linear in the loop momenta.

[79] In summary, if a marked point $z_{i}$ is shifted by a " $B$ cycle," (i) the loop momentum associated to that cycle is shifted by $k_{i}$ and (ii) the Abelian differential of the third kind has nontrivial monodromy. These two effects combine precisely to achieve homology invariance. See the very clear discussion for the two-loop five-point amplitude in Ref. [80], where the objects $g_{i, j}^{I}$ relate to $\omega_{3,4}\left(z_{1}\right)$ as $\omega_{3,4}\left(z_{1}\right)=\left(g_{1,3}^{I}-g_{1,4}^{I}\right) \omega_{I}\left(z_{1}\right)$.

[80] E. D'Hoker, C. R. Mafra, B. Pioline, and O. Schlotterer, Two-loop superstring five-point amplitudes. Part I. Construction via chiral splitting and pure spinors, J. High Energy Phys. 08 (2020) 135.

[81] E. D'Hoker and D. H. Phong, Lectures on two loop superstrings, Conf. Proc. C 0208124, 85 (2002).

[82] E. D'Hoker and D. H. Phong, Two loop superstrings. 1. Main formulas, Phys. Lett. B 529, 241 (2002).

[83] E. D'Hoker and D. H. Phong, Two loop superstrings. 2. The chiral measure on moduli space, Nucl. Phys. B636, 3 (2002).

[84] E. D'Hoker and D. H. Phong, Two loop superstrings. 3. Slice independence and absence of ambiguities, Nucl. Phys. B636, 61 (2002).

[85] E. D'Hoker and D. H. Phong, Two loop superstrings 4: The cosmological constant and modular forms, Nucl. Phys. B639, 129 (2002).

[86] E. D'Hoker and D. H. Phong, Two-loop superstrings. V. Gauge slice independence of the N-point function, Nucl. Phys. B715, 91 (2005).

[87] S. Tsuyumine, On siegel modular forms of degree three, Am. J. Math. 108, 755 (1986).

[88] E. Witten, The super period matrix with ramond punctures, J. Geom. Phys. 92, 210 (2015).

[89] And the nonvanishing of another modular form called $\Sigma_{140}$ in the classical Ref. [90].

[90] J. Igusa, Modular forms and projective invariants, Am. J. Math. 89, 817 (1967).

[91] One may also ask why a sum with

$$
S_{\delta}\left(z_{1}, z_{3}\right)^{2} S_{\delta}\left(z_{2}, z_{4}\right)^{2}+S_{\delta}\left(z_{1}, z_{4}\right)^{2} S_{\delta}\left(z_{2}, z_{3}\right)^{2}
$$

is absent from our result, since it has the correct symmetries. It turns out that this sum gives precisely twice the sum (24), at least in the degeneration limit (we expect this to hold beyond the limit too).

[92] S. Grushevsky and R. Salvati Manni, The Vanishing of two-point functions for three-loop superstring scattering amplitudes, Commun. Math. Phys. 294, 343 (2010).

[93] M. Matone and R. Volpato, Superstring measure and nonrenormalization of the three-point amplitude, Nucl. Phys. B806, 735 (2009).

[94] M. B. Green and P. Vanhove, Duality and higher derivative terms in M theory, J. High Energy Phys. 01 (2006) 093.

[95] N. Berkovits, Super Poincare covariant quantization of the superstring, J. High Energy Phys. 04 (2000) 018

[96] N. Berkovits, Covariant quantization of the super particle using pure spinors, J. High Energy Phys. 09 (2001) 016.
[97] N. Berkovits, ICTP lectures on covariant quantization of the superstring, ICTP Lect. Notes Ser. 13, 57 (2003).

[98] N. Berkovits, Multiloop amplitudes and vanishing theorems using the pure spinor formalism for the superstring, J. High Energy Phys. 09 (2004) 047.

[99] E. D'Hoker, M. Gutperle, and D. H. Phong, Two-loop superstrings and S-duality, Nucl. Phys. B722, 81 (2005).

[100] E. D'Hoker and M. B. Green, Zhang-Kawazumi invariants and superstring amplitudes, arXiv:1308.4597.

[101] E. D'Hoker, M. B. Green, B. Pioline, and R. Russo, Matching the $D^{6} R^{4}$ interaction at two-loops, J. High Energy Phys. 01 (2015) 031.

[102] E. D'Hoker, C. R. Mafra, B. Pioline, and O. Schlotterer, Two-loop superstring five-point amplitudes. Part II. Low energy expansion and S-duality, J. High Energy Phys. 02 (2021) 139.

[103] A. Guerrieri, J. Penedones, and P. Vieira, Where is String Theory? Phys. Rev. Lett. 127, 081601 (2021).

[104] A. Tsuchiya, More on one loop massless amplitudes of superstring theories, Phys. Rev. D 39, 1626 (1989).

[105] D. M. Richards, The one-loop five-graviton amplitude and the effective action, J. High Energy Phys. 10 (2008) 042.

[106] A. G. Tsuchiya, On the pole structures of the disconnected part of hyper elliptic g loop M point super string amplitudes, arXiv:1209.6117.

[107] M. B. Green, C. R. Mafra, and O. Schlotterer, Multiparticle one-loop amplitudes and S-duality in closed superstring theory, J. High Energy Phys. 10 (2013) 188.

[108] C. R. Mafra and O. Schlotterer, One-loop superstring sixpoint amplitudes and anomalies in pure spinor superspace, J. High Energy Phys. 04 (2016) 148.

[109] C. R. Mafra and O. Schlotterer, Towards the n-point oneloop superstring amplitude. Part I. Pure spinors and superfield kinematics, J. High Energy Phys. 08 (2019) 090.

[110] C. R. Mafra and O. Schlotterer, Towards the n-point oneloop superstring amplitude. Part II. Worldsheet functions and their duality to kinematics, J. High Energy Phys. 08 (2019) 091.

[111] C. R. Mafra and O. Schlotterer, Towards the n-point oneloop superstring amplitude. Part III. One-loop correlators and their double-copy structure, J. High Energy Phys. 08 (2019) 092.

[112] S. Grushevsky, Superstring scattering amplitudes in higher genus, Commun. Math. Phys. 287, 749 (2009).

[113] S. L. Cacciatori, F. Dalla Piazza, and B. van Geemen, Genus four superstring measures, Lett. Math. Phys. 85, 185 (2008).

[114] R. Salvati-Manni, Remarks on Superstring amplitudes in higher genus, Nucl. Phys. B801, 163 (2008).

[115] A. Morozov, NSR Superstring Measures Revisited, J. High Energy Phys. 05 (2008) 086.

[116] S. Grushevsky and R. Salvati Manni, The superstring cosmological constant and the Schottky form in genus 5, Am. J. Math. 133, 1007 (2011).

[117] M. Matone and R. Volpato, Getting superstring amplitudes by degenerating Riemann surfaces, Nucl. Phys. B839, 21 (2010).

[118] M. Matone and R. Volpato, Higher genus superstring amplitudes from the geometry of moduli space, Nucl. Phys. B732, 321 (2006). 
[119] Z. Bern, J. J. M. Carrasco, L. J. Dixon, H. Johansson, and R. Roiban, Simplifying multiloop integrands and ultraviolet divergences of gauge theory and gravity amplitudes, Phys. Rev. D 85, 105014 (2012).

[120] Z. Bern, J. J. Carrasco, W.-M. Chen, H. Johansson, and R. Roiban, Gravity Amplitudes as Generalized Double
Copies of Gauge-Theory Amplitudes, Phys. Rev. Lett. 118, 181602 (2017).

[121] Z. Bern, J. J. M. Carrasco, W.-M. Chen, H. Johansson, R. Roiban, and M. Zeng, Five-loop four-point integrand of $N=8$ supergravity as a generalized double copy, Phys. Rev. D 96, 126012 (2017). 